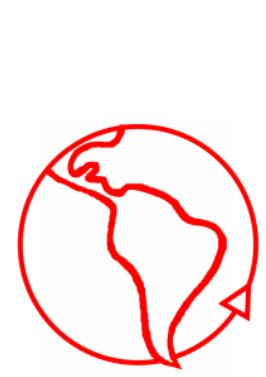

\title{
Hacia la delimitación de un marco teórico conceptual para la investigación evaluativa
}

\author{
Towards the Delimitation of a Conceptual Theoretical Framework for \\ Evaluation Research
}

Gabriela Azócar

Departamento de Sociología, Universidad de Chile, Chile

\begin{abstract}
RESUMEN
Los distintos enfoques aplicados a la evaluación de las políticas públicas se sitúan generalmente, en un marco conceptual de carácter técnico. Esto ha significado que las políticas públicas son entendidas como procesos secuenciales que deben ser analizados por etapas y en función de definiciones unificadoras de problemas de intervención. Tales enfoques, sin embargo, simplifican y limitan el análisis de estos complejos procesos de toma de decisiones. Al trabajar a partir de los conceptos de coordinación descentralizada y diferenciación contextual, es posible identificar distintas dimensiones involucradas en los procesos de intervención pública a la vez que las formas como estas se vinculan y coordinan en su implementación. Entender las políticas públicas más allá de su dimensión política y reconociendo la contraposición de intereses y expectativas como factores determinantes de sus resultados, obliga a repensar la investigación y evaluación de las políticas públicas, alejándose de las estrategias más tradicionales que se centran en enfoques de carácter técnico.
\end{abstract}

PAlabras Clave: Políticas Públicas; Evaluación; Coordinación; Diferenciación Contextual; Estado

\section{ABSTRACT}

The different approaches to public policy evaluation are usually situated in a framework of a technical nature. This has meant that public policies are understood as sequential processes that must be analyzed in stages and in function of unifying definitions of the intervention problems. Such analysis ap proaches, however, simplify and limit the analysis of these complex decision making processes. By working from the concepts of decentralized coordination and contextual differentiation, we can identify different dimensions involved in the processes of public intervention, as well as the ways on which these are linked and coordinated in its implementation. Understanding public policies beyond their political dimension and recognizing the conflict of interests and expectations as determinants of outcomes; force us to rethink the research and evaluation of public policies, moving away from more traditional strategies focused on technical approaches.

KEYWORDS: Public Policies; Evaluation; Coordination; Contextual Differentiation; State

\section{INTRODUCCIÓN}

Entender la evaluación de políticas públicas como una forma de investigación implica reflexionar sobre las herramientas analíticas usualmente utilizadas para el desarrollo de estos procesos. Esto, significa avanzar en la delimitación de referentes interpretativos que permitan comprender cómo los resultados obtenidos de una evaluación se vinculan con

REVISTA MAD - UNIVERSIDAD DE CHILE

Revista del Magíster en Análisis Sistémico Aplicado a la Sociedad

Facultad de Ciencias Sociales, Departamento de Antropología.

Universidad de Chile

www.revistamad.uchile.cl 
nociones más amplias, asociadas a las características del orden y cambio social en la sociedad moderna. Para ello, resulta necesaria la elaboración de marcos teóricos y conceptuales que generen modelos de análisis e interpretación amplios de los resultados de una evaluación de política pública. Por otro lado, se espera que estos mismos referentes de análisis posibiliten la observación de cómo los componentes involucrados en el diseño e implementación de una política afectan de diferentes formas sus resultados, haciendo que lo que inicialmente de ideó como plan de intervención se transforme progresivamente en un distinto modo de concebir el problema y la forma de abordarlo.

Lo que se propone es vincular el análisis de las políticas públicas con los procesos de transformación que la forma de operar del Estado ha experimentado en función del aumento de complejidad que subyace a la sociedad moderna. A partir de ello, se identificarán dos principales modelos de operación estatal, utilizando como referente descriptivo el concepto de coordinación social. Posteriormente, se propondrá situar el análisis de las políticas públicas sobre la base de los conceptos coordinación descentralizada y diferenciación contextual, estableciendo la manera en que ambos se vinculan en la comprensión de cómo se estructuran y operan los procesos de toma de decisiones caracterizados por la vinculación de diversos referentes a sistemas, intereses y expectativas. Desde este marco de referencia se reflexionará sobre la necesidad de repensar los modelos comúnmente utilizados para la evaluación de políticas públicas, estableciendo puntos de quiebre con aquellas formas de análisis generalmente centradas en aspectos de carácter técnico. A partir de los resultados de esta discusión se invita a generar nuevas formas de observación y estrategias de análisis y evaluación de los procesos y resultados asociados a la implementación las políticas públicas hoy.

1. ANTECEDENTES: SOCIEDAD MODERNA, ESTADO Y TRANSFORMACIONES DE LAS POLÍTICAS PÚBLICAS

Comprender la política pública hoy, implica comprender cómo el Estado ha ido transformando sus lógicas de operación, adaptándose con ello a la estructura compleja asociada al orden social representado por la modernidad. Desde tal perspectiva, podemos decir que las políticas públicas, entendidas como una forma de operar del Estado, así como dinámicas de coordinación social, han progresivamente evolucionado desde modelos de operación de carácter jerárquico y centralizado hacia lo que se definirá 
como modelos de coordinación descentralizada y de jerarquía relativa. Al respecto se ha planteado que, para profundizar en las repercusiones del aumento de complejidad social sobre el Estado, es necesario comprender y definir como este opera a través del diseño e implementación de políticas públicas considerando que, a través del análisis de tales procesos y decisiones, es posible observar la manera en que ha reaccionado ante los sucesivos cambios que la modernidad le ha impuesto respecto de su relación con la sociedad.

Para comprender este proceso de evolución, es relevante señalar que las políticas públicas, como objeto de estudio y análisis, adquieren progresiva relevancia a partir del siglo XIX, periodo en el cual la producción de conocimiento científico sobre los problemas sociales pasó a constituir un requisito para la toma de decisiones de carácter público. Durante este periodo, la definición de políticas públicas empieza a abarcar cada una de las distintas dimensiones de la vida social (Parsons 2007). Manteniendo esta orientación, a principios del siglo $X X$, la generación de conocimiento sobre los problemas políticos, sociales y económicos sobre los cuales el Estado se ve llamado a intervenir, se realiza bajo una perspectiva de carácter positivista, en la que predominan los modelos de investigación cuantitativo y conductista. La aspiración a la objetividad y neutralidad valórica que caracterizan a esta forma de producción de conocimiento, implica que la investigación ligada a la política pública se centre en el análisis de datos y variables numéricas que, vinculados de manera causal, pretenden explicar el origen y consecuencias de los problemas investigados, con el fin de identificar soluciones concretas a tales situaciones. En este periodo y desde tal perspectiva, los fines y posiciones normativas asociadas a las decisiones de política pública no son materia de discusión ni son considerados como información relevante para el diseño de la política pública (Pérez 2005). Esto no quiere decir, sin embargo, que la formulación de política pública en este tiempo carezca de una orientación normativa, muy por el contrario, lo que se observa es que ante el primado moral del bien común y la idea de unidad representadas en figuras tales como el Estado nación, se crea la ilusión de una ética fundamental que guía el proceso de decisiones políticas y que, como tal, no tiene posibilidades de ser cuestionada. En tal contexto, el modelo positivista de producción de conocimiento resulta funcional a la necesidad de buscar respuestas a problemas sociales que obstruyen el principio general del bien común, en tanto este no da cabida al análisis de posiciones valóricas divergentes. Esta oscilación entre referencias científicas y normativas no impide, sin embargo, que las 
políticas públicas durante este periodo sean comunicadas como decisiones asumidas por el Estado y que, por ende, predomine en ellas su carácter político y su sentido jerárquico.

Es posible decir que los contextos diferenciados de producción de políticas públicas durante el siglo XIX se caracterizan por una marcada reducción de complejidad en términos de homogenización. Esto se observa en que los problemas sociales se estudian desde una perspectiva científica pero se definen en decisiones de carácter político. La toma de decisiones se centraliza en la figura del estado y las expectativas de los agentes y actores sociales a los que hacen referencia los problemas públicos se reducen y simplifican bajo el principio normativo del bien común. De ello se deduce que la estrategia de coordinación de estos distintos elementos esté orientada políticamente en función del principio de control.

Un segundo momento del proceso evolutivo de las políticas públicas puede ser identificado a mediados del siglo XX. En un escenario marcado por las crisis políticas y económicas de la post guerra, la consolidación del sistema capitalista y el aceleramiento de los procesos de diferenciación social, el Estado comienza a ser sobre-demandado ante una cada vez más amplia diversidad de exigencias y necesidades que reflejan el aumento de complejidad que caracteriza a la sociedad moderna. Este nuevo escenario implica que la dimensión ética de la definición de políticas públicas adquiera especial relevancia, dado el carácter de los problemas sociales que en este periodo se configuran como foco de la intervención estatal. Luego de dos guerras mundiales, los principios asociados a la noción de derechos humanos, pasan a ser el referente articulador de los fines políticos, a lo que se suma la creciente preocupación por el incremento en las desiguales posibilidades de acceso y participación en las esferas de la sociedad y, por ende, en las dificultades que esto conlleva en asegurar condiciones de vida básicas para el desarrollo de las personas. Lo que se perseguía entonces era reducir los niveles de inseguridad que se derivaban de esta mayor complejidad social. Se puede decir que en este segundo periodo es posible observar una serie de cambios en relación con la etapa anterior, en tanto la orientación normativa de las políticas públicas adquiere mayor predominancia en su definición, a la vez que se empieza a reconocer una mayor diversidad de actores y expectativas relacionadas con problemas sociales de carácter público.

El Estado de bienestar en Europa así como el Estado desarrollista en América Latina, constituyeron formas en las que se reaccionó ante la exigencia social por solucionar las distintas $y$, no pocas veces, agudas condi- 
ciones de exclusión social que caracterizaron el desarrollo de las sociedad moderna a partir del periodo de las post guerras. Ambos casos constituyen modelos centralizados de intervención, donde el Estado figura como el actor responsable y principal en el diseño de soluciones de carácter público a los nuevos y crecientes problemas sociales y económicos de la sociedad. En esta etapa, la esfera política sigue entonces predominando en la comunicación de las políticas públicas.

La provisión de servicios sociales en la búsqueda de asegurar un estándar de vida general y el fomento al desarrollo industrial interno no fueron, sin embargo, medidas capaces de responder eficientemente ante la constante emergencia de problemáticas y demandas impuestas por una acentuada diversificación de las formas de exclusión social. Se puede decir que el cuestionamiento a la democracia en su capacidad de respuesta ante los problemas la sociedad moderna es una consecuencia del relativo fracaso con que se ha sentenciado a estos modelos de intervención estatal (Willke 2007).

Este conjunto de factores sociales y normativos tuvieron evidentes efectos sobre la forma de entender y abordar el diseño e implementación de las políticas públicas a partir de las últimas décadas del siglo pasado, lo que nos permite identificar un tercer periodo de análisis. Los principales cambios que en esta nueva etapa se observan, muestran que, por una parte, el foco de atención dejó de estar puesto en la identificación de soluciones a partir de datos fácticos sobre los problemas de orden público y que, por otro, la dimensión normativa del diseño de políticas públicas empezó a ser entendida en función de la necesidad de debatir en torno a los distintos intereses y posiciones valóricas que configuran alternativas de solución a los problemas que se pretende resolver. Las políticas públicas modernas pasaron a ser entendidas entonces como cadenas de decisiones en las que esta diversidad de perspectivas logra vincularse. En este nuevo escenario, el diseño e implementación de políticas públicas se caracteriza por delimitar espacios complejos y diferenciados de toma de decisiones, cuya orientación simbólica deja de ser exclusivamente política, en tanto oscila con mayor intensidad que en periodos anteriores entre referentes de carácter económico, científico y normativo. Esta nueva forma de conducción de la toma de decisiones relativas a políticas públicas ha sido denominada y definida desde distintas perspectivas.

Un enfoque de carácter descriptivo lo encontramos el análisis de las policy networks, el que ha sido ampliamente desarrollado por distintos autores. Esta perspectiva surge del análisis de políticas y programas públi- 
cos realizados en distintos países europeos a partir de la década de 1990, con lo que se ha pretendido describir las nuevas formas de operar en el diseño e implementación de políticas públicas de carácter complejo (Börzel 1998; Kisby 2007). Desde este enfoque se plantea que las políticas públicas se desarrollan a partir de la vinculación descentralizada entre actores públicos de carácter estatal y actores privados que se reúnen en función de un propósito común, configurando con ello lo que se denomina como policy networks (Scharp \& Mohr 1994; Börzel 1998; Messner 1999; Chaqués 2006). Se señala que los actores que participan de este tipo de redes representan intereses particulares que, como tales, pueden encontrarse en conflicto, factor que constituye una de las principales características de estas estrategias de diseño e implementación de políticas públicas. Cabe señalar que la referencia al sistema económico adquiere una particular relevancia en este enfoque, lo que se refleja en el lenguaje que se utiliza para describir cómo se enfrentan los conflictos de intereses y expectativas entre actores. Se plantea que para ello se establecen interacciones horizontales de negociación e intercambio mediante las cuales se distribuyen los costos y beneficios que se obtienen como resultado de las decisiones colectivas que la red adopta (Kjaer 2008).

Por otro lado, la dimensión normativa de estas formas de operación estatal se define como la configuración de procedimientos formales que regulan a sus actores y cuyas normas dotan de legitimidad al proceso de toma de decisiones colectivas, lo que a su vez ratifica a las policy networks como mecanismos de representación (Mayntz 1998). Estos procedimientos delimitan la función y facultades de los participantes en la red sin que con ello estos pierdan su autonomía y vean afectados sus intereses particulares. Con ello se reconoce una dependencia recíproca, que dota de coherencia a las decisiones y acciones que la red adopta en función de la resolución de problemas de carácter público.

Otro de los enfoques utilizados para el análisis de las políticas públicas es el que plantea la noción de gobernanza. Cabe señalar que, si bien este concepto también es de origen europeo, su utilización en el estudio de formas de vinculación entre actores públicos y privados ha sido ampliamente aplicada en contextos latinoamericanos. La noción de gobernanza surge como parte del análisis crítico sobre los resultados de formas jerárquicas y centralizadas de conducción del Estado que comienza a ser desarrollado en la época de las post guerras, cuando los Estados europeos asumen la tarea de dirección del desarrollo social y económico de sus naciones (World Bank 1992). La gobernanza comienza a figurar como un es- 
tilo alternativo de dirección política que se caracteriza por un modo horizontal y descentralizado de operar, con el cual se persigue acreditar al Estado como un agente capaz de articular y responder ante las demandas sociales, en un contexto en el que la democracia empieza a ser cuestionada en relación con su eficiencia. Desde un principio, la noción de gobernanza se encuentra ligada al análisis de las políticas públicas cuando, a partir de los años sesenta, estas empiezan a ser vistas como un objeto particular de estudio, especialmente en el campo de las ciencias políticas y la administración pública. En este período, el énfasis del análisis estaba puesto en la forma de operar de los políticos y legisladores encargados de los procesos de diseño e implementación de las políticas públicas, enfoque que más tarde se traslada al análisis de la capacidad de respuesta de los Estados democráticos ante el aumento de diversidad observado en el surgimiento de nuevas demandas sociales. A partir de ello, la gobernanza adquiere una nueva dimensión y significado, en tanto se transforma en un concepto mediante el cual se da cuenta de las relaciones que el Estado establece con actores privados y organizados de la sociedad. La necesidad de considerar otros sectores de la sociedad en los procesos de toma de decisiones de política pública, se basa en la premisa de que los recursos requeridos para la ejecución de tales políticas se encuentran dispersos entre actores públicos y privados, lo que determina la interdependencia de los intereses del conjunto de ellos (UNDP 1997). Por otro lado, con esto se da cuenta que, para resolver los problemas sociales que son el foco de las políticas públicas, hay que entender que no solo los recursos se encuentran dispersos en la sociedad, sino que también el poder de utilizar estos recursos en función de intervenir aquellas áreas sociales que presentan problemas. A partir del concepto de gobernanza se describe entonces una nueva forma de operar del Estado, donde este figura como el encargado de movilizar y reunir los recursos que se encuentran en su entorno. Con ello se da cuenta de la creciente dependencia del Estado de los recursos y la colaboración de actores no estatales que carecen de poder político (Börzel 1998). Esto hace evidente la necesidad de reconocer e incorporar los distintos intereses de estos actores, a la vez que los de aquellos que hasta ese entonces habían sido vistos solo como beneficiarios o receptores de estas políticas (Aguilar 2009). Se puede decir que, con ello, el estudio de las políticas públicas, a la vez que se aleja de la perspectiva administrativa, se aproxima más al análisis sociológico, en tanto incorpora como factores relevantes el aumento y consecuencias de la diferenciación interna 
de la política, así como la necesidad de transformar su forma de vinculación con el entorno social.

La gobernanza, si bien es semejante en sus elementos, se diferencia del enfoque de análisis basados en la policy netwoks, en tanto pone mayor énfasis en la revisión los principios éticos y valóricos que subyacerían a las interrelaciones y expectativas de los actores sociales que participan de tales procesos. En tal sentido es que la crítica a los modelos jerárquicos y centralizados de conducción estatal de la sociedad, desde la cual surge y se desarrolla el concepto de gobernanza, implica una crítica al principio unificador de bien común sobre el cual se sustentaban normativamente tales modelos. Se plantea entonces que el principio del bien común bloquea la posibilidad de observar, reconocer y validar la existencia de distintas posiciones éticas y valóricas, desde las cuales se construyen expectativas y aspiraciones particulares de bienestar individual. Esto actuaría como un argumento de por qué tales modelos no serían capaces de responder a las demandas de una sociedad moderna, en la que la diversidad de construcciones valóricas de proyectos de vida, constituye parte de su complejidad. Con la idea de gobernanza se tiende entonces a reemplazar el principio del bien común por el postulado sobre el reconocimiento de esta diversidad de valores que se reflejan en los distintos intereses que entran en juego al momento de la vinculación entre actores públicos y privados. Con ello, por ende, se niega la posibilidad de un enfoque común, a la vez que se apela a ideales normativos tales como la colaboración y la deliberación como formas de resolución de las divergencias que surgen del enfrentamiento de intereses diversos.

Podemos decir que el conjunto transformaciones antes descritas han repercutido en que actualmente el análisis de las políticas públicas ha pasado a centrarse en los procesos de decisión a partir de los cuales estas son elaboradas e implementadas. Por otro lado, desde una perspectiva centrada en la conceptualización teórica de la políticas públicas, es posible decir que en la actualidad estas pueden ser abordadas y analizadas como ámbitos particulares de diferenciación, cuya función es la resolución de problemas de carácter público, en los que confluyen, entre otros, la referencia a distintos sistemas sociales, cuya orientación simbólica es la que tematiza los intereses y expectativas de los diversos actores que participan en su definición e implementación. 
2. COORDINACIÓN Y DIFERENCIACIÓN CONTEXTUAL COMO HERRAMIENTAS DE ANÁLISIS PARA LA EVALUACIÓN DE LAS POLÍTICAS PÚBLICAS

Para una mejor comprensión de cómo las políticas públicas se articulan como ámbitos particulares de operación, es necesario recurrir a un concepto central para este tipo de análisis, a saber, el concepto de coordinación. En este marco dicho concepto adquiere particular relevancia, en tanto permite describir cómo se vinculan los sistemas funcionales y aquellos actores que representan los intereses y expectativas que configuran procesos de toma de decisiones mediante las cuales se pretende dar respuesta a problemas y demandas sociales de carácter público. Se propone en este punto una primera clasificación teórica de al menos dos principales modelos de coordinación posibles de ser aplicados en el marco de análisis de las políticas públicas.

Se denominará al primer modelo como coordinación centralizada, concepto que se ajusta a lo planteado por Lechner (2007) en términos de coordinación política, en tanto alude a una estrategia de organización en la que el Estado figura como autoridad encargada de la resolución de los problemas públicos. En este modelo, la referencia al sistema político prima por sobre lo demás. Esto significa que las políticas públicas se definen como decisiones exclusivamente políticas, desprendiéndose de posibles referencias a otras esferas funcionales, tales como la ciencia, la economía o el derecho. En este modelo, el Estado se caracteriza por su escasa vinculación con el entorno. Se trataría, por ende, de un contexto menos complejo en el que el Estado tiende a simplificar las decisiones sobre los problemas de carácter público, en tanto los comunica como problemas políticos.

En este marco y bajo el criterio de control, los intereses de actores que no pertenecen a la esfera del Estado no serían considerados en la planificación de estrategias de intervención, pasando a jugar un rol instrumental, como aportadores de recursos necesarios para la ejecución de decisiones estatales. De ello deriva un modo centralizado de toma de decisiones, según el cual el Estado es quien decide qué estrategias de solución serán adoptadas en base a lo que ha definido como problemas de carácter público. Esto limitaría la capacidad del Estado de anticipar las consecuencias que los intereses de los actores privados, puedan tener sobre sus propias decisiones. Se desprende entonces que el principal recurso de aprendizaje en este modelo de coordinación es el de la propia experiencia, lo que hace que la evaluación de los resultados de políticas públicas previas adquiera especial valoración como fuente de conocimiento. 
Otra característica de la coordinación centralizada es que, desde esta perspectiva de análisis, se entiende que las expectativas normativas de los actores no estatales serían consensuadas en principios unificadores que apelan al bienestar general de la población, obstruyéndose con ello la capacidad de observar diferentes posiciones valóricas. Por ende no se reconoce conflicto, en tanto la definición de los problemas se realiza de manera autoritativa. La estructura de relación entre los actores públicos y privados adoptaría por lo tanto una forma piramidal, en la que el Estado estaría ubicado en la cúspide.

Un segundo modelo de coordinación, que denominaremos como coordinación descentralizada, corresponde a aquel que se ajusta al ordenamiento diferenciado de la sociedad moderna y que se caracteriza por la mayor cantidad de elementos que deben ser vinculados en cada una de sus dimensiones, a la vez que por la horizontalidad de la estructura de relación entre los mismos.

Este modelo se caracteriza por incluir referencias a distintos sistemas de manera oscilante, a partir de variaciones temáticas, en distintos momentos de la definición e implementación de políticas públicas. Correspondería por lo tanto a un contexto de mayor complejidad, aunque la participación de diversas esferas sociales sería solo relativa a determinados momentos de decisión. Esta perspectiva plantea que los problemas públicos pueden ser definidos como políticos, económicos, normativos o científicos, así como las decisiones sobre cómo intervenirlos. Ellos pueden ser observados desde cada uno de estos sistemas y por ende de ello depende la primacía relativa que estos adquieran en su comunicación (Azócar 2009).

En este escenario, los actores públicos y privados participan de los procesos de toma de decisiones sobre política pública, lo que implica que sus distintos intereses son considerados en ellas. El conflicto que deriva de intereses en contraposición es reconocido como un medio de vinculación, en tanto se trabaja colaborativamente en la determinación de acuerdos. Los actores reconocen una relación de mutua dependencia para la consecución de sus propósitos, por ende la toma de decisiones es de carácter descentralizado. La vinculación se orienta al reconocimiento de las diferencias y al paralelismo de intereses.

Por otro lado, desde este enfoque se reconocen distintas expectativas normativas sobre los resultados de los procesos de implementación de las políticas públicas, las que son consideradas como parte de la definición de los problemas públicos y sus estrategias de intervención. La orientación 
normativa de este modelo es el reconocimiento de las diferencias y el trabajo colaborativo en la toma de decisiones. Esto repercute en que la estructura de relación entre los actores públicos y privados sea de carácter horizontal.

Utilizar el concepto de coordinación descentralizada para el análisis de las políticas públicas implica, desde una perspectiva teórica, identificar al menos 3 niveles de referencia que permiten visualizar la forma que estas adquieren: la referencia a sistemas sociales, la referencia a intereses particulares y la referencia a expectativas diferenciadas. La relación entre estas tres dimensiones, si bien parece ser evidente, no resulta simple de plantear, dada la multiplicidad de elementos que las configuran y el hecho de que cada una de ellas alude a espacios particulares de observación que representan distintos niveles de abstracción. Es posible, sin embargo, reconocer que estos niveles de diferenciación social, en su vinculación, conforman contextos particulares de operación social posibles de ser descritos a partir del enlace de sus elementos. Esta combinación de formas de diferenciación ha sido denominada por Mascareño (2011) como "diferenciación contextual", concepto que el autor define como un tipo de formación social que emerge como consecuencia del aumento progresivo de complejidad de la sociedad moderna, delimitando ámbitos específicos de interacción, decisión y orientación simbólica que logran diferenciarse de su entorno, en tanto hacen referencia a temas, intereses y expectativas particulares.

El concepto de diferenciación contextual adquiere especial relevancia como herramienta analítica de la política pública, en tanto permite comprender cómo las transformaciones de las formas de operar del Estado se vinculan con los procesos de aumento de complejidad de la sociedad moderna, en tanto evolucionan progresivamente hacia la combinación de temas, intereses y expectativas diversas. Desde esta perspectiva de análisis, se propone entender las políticas públicas como formas particulares de diferenciación contextual, cuya función es atender aquellos problemas sociales de carácter público que se han constituido como demandas al Estado, pero que se resuelven a partir de la vinculación de diversas esferas y actores.

\section{VOLVER A PENSAR LA EVALUACIÓN}

A partir de los antecedentes y conceptos recién expuestos, se plantea la posibilidad de comprender el proceso dinámico de desarrollo de una 
política pública, lo que actuaría como marco teórico conceptual de referencia para la estructuración de procesos de investigación evaluativa. En la práctica, sin embargo, formas de ordenamiento conceptual como esta no son siempre utilizadas, dado que en el proceso evaluativo prima generalmente el desarrollo de estrategias de carácter técnico que tienden a desagregar el análisis de las políticas públicas en etapas, imputando con ello una lógica de análisis de carácter cuantitativa y lineal que no siempre se ajusta a la complejidad y dinámica de estos procesos.

Si se realiza el ejercicio de pensar cómo opera en la práctica una política pública, para muchos resulta evidente que estas consisten en una cadena secuencial de acciones sobre problemas o áreas que se han identificado como problemáticas y que por ende requieren ser intervenidas. Bajo esta perspectiva de secuencialidad es que el análisis y la evaluación de las políticas públicas opera en función del estudio de fases sucesivas. Las más comunes se refieren al diseño de la política, centrado en la identificación y definición del problema que se espera intervenir y resolver; el proceso de planificación de la política, referido a la ideación de planes de acción concretos, a partir de la gestión de recursos y herramientas de carácter técnico; y finalmente una etapa ejecutiva de implementación de lo que en la etapa anterior se ha proyectado realizar. La evaluación de una política pública se plantea generalmente a partir del análisis de estas etapas, identificando el problema que se pretendía intervenir, analizando qué metas y resultados fueron proyectados $\mathrm{y}$, primordialmente, dando cuenta del modo en que tales propósitos se lograron (Arellano 2010; Cejudo \& Merino 2010; Lahera 2008). Todos quienes han participado de un proceso de evaluación de una política pública saben que, sin embargo, al ajustarse de manera estricta a un esquema secuencial como este, no es posible analizar completamente lo que efectivamente sucede en estos procesos de intervención, los que resultan mucho más complejos que el esquema inicial de diseño, planificación e intervención, con el cual se insiste en empezar a trabajar. Es común que el proceso de evaluación de las políticas públicas devele que el problema que inicialmente se definió como objeto de intervención no sea comprendido de la misma manera por todos los actores involucrados en estos procesos; que lo que se planificó hacer no siempre se hizo, pero que se hicieron más cosas que las que inicialmente se pensaron; y que si bien no todas las metas se cumplieron, las consecuencias no previstas de los procesos de intervención repercuten en resultados diferentes a los proyectados, los cuales generalmente amplían y enriquecen el análisis. Con ello, las conclusiones de la evaluación de una política pública 
generalmente indican la necesidad de considerar nuevas dimensiones del problema en futuros procesos de intervención, nuevas formas de planificarlo y nuevas formas de llevar a cabo los procesos de implementación. Es por ello que, al finalizar una evaluación, los investigadores se quedan con la sensación de que ahora saben cómo se debe idear una intervención y que si sus recomendaciones son consideradas, los programas y acciones venideros resultarán más eficientes y eficaces que los recientemente evaluados. Se quedan con la idea de que la evaluación, como proceso investigativo, ha generado nuevos conocimientos que servirán como antecedentes fundamentales para el diseño de nuevas políticas en la materia. La evaluación de los procesos posteriores, sin embargo, mostrará que nuevamente los problemas, los planes y las formas de implementación que consideraron sus recomendaciones, terminan en resultados diferentes de los esperados. Quienes han trabajado en evaluación de políticas saben que esto es común pero no se dejan afectar por ello, dado que saben la riqueza investigativa que supone llevar a cabo un proceso evaluativo de esta naturaleza. Llama la atención, sin embargo, la persistencia en seguir utilizando como marco de referencia las mismas etapas secuenciales antes señaladas, en la forma en cómo se estructura y piensa la evaluación, aún a sabiendas de que tales etapas no son necesariamente sucesivas y que lo que ellas muestran es solo una parte de un proceso más complejo de analizar.

Retomaremos la idea antes planteada de entender las políticas públicas a partir del concepto de coordinación descentralizada y los tres niveles de referencia que, desde la noción de diferenciación contextual, se vinculan en la conformación de una política pública. Esto supone, en cierta medida, superar la lógica de la secuencialidad y complejizar la forma de comprender a las políticas públicas como objeto de investigación. Si se piensan las políticas públicas en función del concepto de coordinación tenemos que identificar aquellos elementos que, en vinculación, nos permiten observar conceptualmente como estas se configuran, como operan y por ende como pueden ser evaluadas. Para ello resulta relevante adscribir a aquellas corrientes de análisis de las políticas públicas que señalan que estas pueden ser entendidas como una cadena de decisiones vinculadas, adoptadas por actores de diferente naturaleza, es decir actores públicos y privados, que participan directa e indirectamente en su implementación. Desde esta perspectiva, se puede señalar que las políticas públicas pueden ser entendidas como procesos decisionales dinámicos, más que como una sucesión de etapas, a la vez que como una vinculación de referentes que 
se encuentran contenidos y representados por los diversos actores que en ellas participan.

En base a lo anterior, lo que se propone es que el proceso investigativo y la evaluación de las políticas públicas debe considerar 3 niveles de referencia en el análisis. En primer lugar, está la referencia a sistemas sociales específicos, a partir de la cual distintas perspectivas de conocimiento influyen, tanto en las decisiones sobre la definición del problema de la política, como en las decisiones sobre cómo ese problema será abordado. Esto significa entender que las políticas públicas responden a diferentes orientaciones simbólicas y que por ende no pueden ser entendidas exclusivamente en función de una orientación netamente política. Una política pública oscila entre referencias políticas, económicas, normativas y científicas, las que pueden predominar sobre las demás en distintos momentos del proceso de toma de decisiones que las configuran. Esto implica que, en un proceso de investigación evaluativa, no es posible definir desde una única perspectiva el problema de intervención y que, por ende, en distintos momentos de la toma de decisiones, nos encontraremos con énfasis distintos sobre cómo concebir y abordar el objeto de la política. La definición del problema por lo tanto se irá reconfigurando continuamente, lo que implica que no puede ser situada en una única etapa del proceso. Esto implica romper con la lógica tradicional de la evaluación, la que señala como necesaria la identificación inicial del problema de intervención para, a partir de este, delimitar los niveles de logro alcanzados durante el desarrollo de una determinada política pública. Este quiebre alude a la necesidad de reconocer que el problema con que se inició la intervención no es necesariamente el mismo problema con el que se culminó este proceso; que los distintos actores involucrados en la ejecución de la intervención no coinciden en la forma en que definen y significan el problema; y que, principalmente, los sentidos y fundamentos del problema fueron cambiando durante los distintos momentos en que fue abordado.

Se debe considerar el nivel de influencia que tienen formas alternativas de comprender el problema sobre los resultados e impactos obtenidos de una política pública. Si bien en la definición del problema puede predominar un sistema de argumentos por el cual se ha optado y mediante el cual se ha comunicado lo que se entiende por este, esto no implica que otras formas de concebirlo no hayan influido en la forma en cómo fue abordado. Ese tipo de influencias pudieran ser explicativas de posibles incongruencias en la forma de operar de la política o bien pudieran repre- 
sentar estrategias de adaptación ante la emergencia de factores y situaciones que previamente no fueron considerados.

La definición de los problemas públicos debe ser analizada teniendo en cuenta la diversidad de esferas sociales a las que refieren, no pueden ser entendidos por lo tanto desde una perspectiva univoca. En el momento de la evaluación se debe reconocer, por ejemplo, cómo el problema sobre el cual se ha trabajado se orienta al aseguramiento de derechos, a la reparación de un daño moral o bien a la obstaculización de la inclusión en esferas funcionales de la sociedad. Las diferentes lógicas que hay detrás de cada una de estas referencias determinarán cómo se configuran las estrategias de intervención mediante las cuales las políticas públicas se implementan, lo que significa que ninguna de ellas puede ser descartada como parámetro de evaluación

En segundo lugar, encontramos el nivel de referencia representado por los intereses involucrados en la toma de decisiones sobre política pública, a partir de los cuales es posible identificar cómo distintos tipos de actores se vinculan y participan en la implementación de una política pública. En este punto podemos hablar de intereses particulares y colectivos que, a su vez, se vinculan con orientaciones políticas, económicas, normativas y científicas, enlazándose de este modo con el nivel de referencia relativo a los sistemas. Esto, a su vez, permite analizar los recursos aportados por estos distintos actores en la implementación de la política pública, en tanto delimitan y determinan los aportes económicos, técnicos y políticos con los que cada uno contribuye y con los que determinan su modo de participación. Cabe señalar un elemento relevante para el proceso de evaluación de la política pública, relacionado con la identificación y reconocimiento de esta pluralidad de intereses: el conflicto que puede derivar de su contraposición. Reconocer tales tipos de conflicto implica develar una de las formas en la que la complejidad opera, entendiendo que el consenso no es la única manera de hacerlo. Al respecto, cabe señalar que un proceso de investigación evaluativa requiere determinar cómo este tipo de tensiones logran o no ser resueltas y las consecuencias que esto supone. Esto implica además dejar de pensar el conflicto como un obstáculo e introducirlo en el análisis evaluativo como un factor determinante en la forma y contenido del proceso de toma de decisiones.

La evaluación de una política pública implica identificar el grado de influencia que los intereses de distintos actores pueden ejercer sobre la forma en que se desarrolla la intervención. Identificar estos distintos niveles de influencia y los momentos en que estos se ejercen dentro de la ca- 
dena de toma de decisiones implica, para un proceso evaluativo, un más complejo y preciso tratamiento de las fuentes de información con las que se trabaja.

En tercer lugar, podemos identificar el nivel de referencia relativo a las expectativas que, desde las esferas y actores participantes en el proceso de desarrollo de las políticas públicas, se configuran en función de los rendimientos o beneficios que estos proyectan como resultado de la intervención. Este nivel resulta especialmente relevante en un proceso de evaluación, si consideramos que alude a los resultados esperados desde distintas posiciones e intereses vinculados a la toma de decisiones, los que deben ser considerados en su diversidad entendiendo además que estos determinaran los niveles de satisfacción de los distintos actores participantes. Esto quiere decir que no es posible evaluar los resultados de una política pública considerando solo la resolución del problema como la principal expectativa de logro. Por otro lado, implica entender que si bien no todas las diferentes expectativas que se generan en función de un proceso de intervención pueden ser resueltas, estas constituyen los criterios evaluativos con los que los involucrados y beneficiarios operan y responden cuando se produce el levantamiento de la información de una investigación evaluativa, lo que significa que desde posiciones de observación diferentes obtendremos perspectivas diferenciadas y contrapuestas sobre el desarrollo de una política pública. La labor del evaluador será ponderar estas diferentes lógicas y proyecciones en función de su influencia sobre los resultados alcanzados.

Se debe considerar que la existencia de distintas expectativas sobre los resultados de una política determinará el nivel de satisfacción que los actores participantes comuniquen al momento de la evaluación, lo que afectará nuestras interpretaciones sobre la estimación de los logros alcanzados y la identificación de factores facilitadores y obstaculizadores del proceso. Lo que se sugiere al respecto es considerar que el cumplimiento o no de una expectativa es un indicador más de los resultados y consecuencias del proceso de intervención. La evaluación de las expectativas es uno de los elementos centrales para la estimación de los residuos, entendidos estos como una medida del error entre lo proyectado y lo obtenido, lo que por lo tanto devela aquel espacio complejo de resultados que pueden ser considerados como consecuencias no previstas de la intervención 
REFLEXIONES FINALES

A modo de resumen, en base a lo anterior podemos señalar que, desde la perspectiva conceptual recién propuesta, elementos relevantes de resaltar y que deben ser consideradas al momento de la evaluación de las políticas públicas son:

- Es necesario comprender que las políticas públicas no se enmarcan exclusivamente en la esfera política, en tanto refieren a esferas simbólicas diversas.

- Dependen de los aportes de distintos actores y estos están determinados por la configuración de sus intereses, los que pueden entrar en conflicto. El conflicto, por lo tanto, no puede ser entendido como un obstáculo, dado que es un factor determinante de los resultados de la intervención.

- Los resultados pueden ser evaluados de manera diferenciada según el nivel de logro definido, desde la construcción de distintas expectativas.

Tener en cuenta lo anterior implica comprender de mejor manera por qué los resultados de los procesos de evaluación resultan altamente complejos y se distancian tanto de lo que inicialmente se propone como marco de referencia. Esto implica además asumir una posición teórica particular en relación con la noción de proyección que subyace a los procesos evaluativos, en tanto, desde esta perspectiva, la proyección que realicemos de los resultados esperados debe ser entendida como una construcción que se lleva a cabo en base a la disposición de conocimientos y valoraciones situados en el momento de decidir y planificar las acciones de política pública. Se debe considerar que siempre se trabajará con información incompleta, dado que no es posible considerar como parte de ello los eventos contingentes que tendrán cabida en el futuro y que sin duda afectarán los procesos y resultados que serán analizados en el momento de la evaluación. En tal sentido, la evaluación implica medir no solo lo que la política pública ha proyectado, implica dimensionar y relevar aquel espacio residual entre ese diseño de futuro y lo que posteriormente se observa como resultado, asumiendo la complejidad del error como uno de los elementos centrales del análisis.

Trabajar a partir de los conceptos coordinación descentralizada y diferenciación contextual implica entender que en los distintos momentos 
asociados a la toma de decisiones, distintos sistemas, intereses y expectativas irán primando por sobre los demás y que por lo tanto no habrá un único centro decisional durante este proceso. De ahí el interés en definir esta forma de coordinación como coordinación de jerarquía relativa. Esto por dos motivos: la estructuración jerárquica asociada a la política pública no ha sido y no puede ser del todo superada al momento de comprender, investigar y evaluar estos procesos. De ello deriva que la referencia más común sobre quién es el responsable del diseño e implementación de las políticas públicas sea el Estado como actor y con ello el sistema político como sistema funcional de referencia. Por más actores y sistemas que estén involucrados en un proceso de toma de decisiones sobre política pública, el Estado y la política tienden a ser a quienes se dirige y exige la responsabilidad del proceso, lo que repercute en que estos referentes particulares tiendan a ocupar con mayor frecuencia una posición de jerarquía. Esto, sin embargo, no significa que en distintos momentos de la cadena de decisiones otros referentes y actores no puedan ascender a dicha posición. Coordinación de jerarquía relativa alude al reconocimiento de la diversidad de referentes y participantes involucrados en estos procesos, a la vez que a reconocer que en la dinámica de la cadena de decisiones estos pueden primar por sobre otros, es decir ocupar una posición principal relativa a la relevancia de la esfera de conocimiento que se está representando, al valor de la contribución que desde esa posición se realiza y a la preeminencia de la proyección que subyace a sus expectativas.

La evaluación, entendida como proceso de investigación, requiere de este tipo de referentes conceptuales que le permitan situarse como un mecanismo de observación externa que sea capaz de reproducir su propia forma de observar y comprender la política pública como objeto de estudio. RM

BIBLIOGRAFÍA

Aguilar, L. F. (2009). Gobernanza y gestión pública. México D.F.: FCE.

Arellano, D. (2010). El enfoque organizacional en la política y la gestión pública: entendiendo a las organizaciones gubernamentales. En G. Cejudo \& M. Merino (Eds.), Problemas, decisiones y soluciones. Enfoques de política pública (pp. 3646). México D.F.: FCE, CIDE.

Azócar, G. (2009). Del control a la coordinación: Las transformaciones normativas del Estado. Tesis para optar al grado de Magíster en Gobierno y Sociedad. Santiago de Chile: Universidad Alberto Hurtado. 
Börzel, T. (1998). Organizing Babylon - On the Different Conceptions of the Policy Networks. Public Administration, 76(2), 253-273.

Cejudo, G. \& Merino, M. (Eds.) (2010). Problemas, decisiones y soluciones. Enfoques de política pública. México D.F.: FCE, CIDE.

Chaqués, L. (2006). Redes de políticas públicas. Madrid: Centro Investigaciones Sociológicas.

Jobert, B. (2004). Estado, sociedad y políticas públicas. Santiago de Chile: LOM.

Kisby, B. (2007). Analysing Policy Networks. Towards an Ideational Approach. Policy Studies, 28(1), 71-90.

Kjaer, P. (2008). Between Governing and Governance: On the Emergence, Function and Form of Europe's Post-national Constellation. Florence: Florence European University Institute. Department of Law.

Lahera, E. (2008). Introducción a las políticas públicas. Santiago de Chile: FCE.

Lechner, N. (2007). El estado en el contexto de la modernidad. En N. Lechner, Obras Escogidas (pp. 387-406). Santiago de Chile: LOM.

Mascareño, A. (2011). Sociología de la intervención: Orientación sistémica contextual. Revista Mad, 25, 1-33.

Merino, M. (2010). La importancia de la ética en el análisis de las políticas públicas. En G. Cejudo \& M. Merino (Eds.), Problemas, decisiones y soluciones. Enfoques de política pública (pp. 27-34). México D.F.: FCE, CIDE.

Messner, D. (1999). La transformación del estado y la política en el proceso de modernización. Nueva Sociedad, 163, 71-91.

Parsons, W. (2007). Políticas públicas: Una introducción a la teoría de la práctica del análisis de políticas públicas. México D.F.: FLACSO.

Pérez, M. (2005). El estudio de las políticas públicas. En M. Pérez (Ed.), El análisis de las políticas públicas (pp. 51-71). Granada: EUG.

Scharp, F. \& Mohr, M. (1994). Efficient Self-Coordination in Policy Networks. A Simulation Study. Köln: Max-Planck-Institut für Gesellschaftsforschun gMPIFG Discussion Paper 94/1.

UNDP. (1997). Reconceptualising Governance. Discussion paper 2. New York: Management Development and Governance Division Bureau for Policy and Programme Support.

Willke, H. (2007). Capacidad de rendimiento del estado y la necesidad de nuevas formas de governance. Persona y Sociedad, 21(2), 9-16.

World Bank. (1992). Gobernance and Development. Washington D.C: The World Bank.

\section{FUENTES DE INTERNET}

Mayntz, R. (1998). Nuevos desafíos en la teoría de la gobernanza. Consultado en marzo 2009 desde: http://latinamerica.dpi.org/documents/Mayntz 000.pdf

SOBRE LA AUTORA

Gabriela Azócar de la Cruz es profesora del Departamento de Sociología de la Universidad de Chile. Magíster en Gobierno y Sociedad por la Universidad Alberto Hurtado, 
Chile y candidata a Doctora en Sociología por la misma universidad. Entre sus áreas de especialización se encuentran: Políticas Públicas, Metodología de la Investigación Social, Sociología Política. Entre sus últimas publicaciones se destacan: La observación de las políticas públicas como espacios de vinculación de complejidad (2013), Coordination as a Mechanism of Risk Observation (2009), Del origen del derecho internacional público a la discusión sobre su constitucionalización (2008).

\section{CONTACTO}

Facultad de Ciencias Sociales, Universidad de Chile

Departamento de Sociología

Capitán Ignacio Carrera Pinto 1045, Nuñoa

Santiago, Chile

F: +56 229787764

gazocarde@u.uchile.cl

Recibido: Diciembre 2013

Aceptado: Marzo 2014 